\title{
Time Over Threshold as a measure of energy response of plastic scintillators used in the J-PET detector
}

\author{
Sushil Sharma on behalf of the J-PET Collaboration \\ ${ }^{1}$ The Marian Smoluchowski Institute of Physics, Jagiellonian University, Lojasiewicza 11, 30-348 \\ Krakow, Poland
}

\begin{abstract}
The Jagiellonian Positron Emission Tomograph (J-PET) is a multipurpose detector being developed to provide an economical alternative of commercially available PETs as well as to perform the tests on the discrete symmetries and entanglement. It is composed of 192 plastic scintillators axially arranged in three cylindrical layers. In the framework of J-PET detector, TimeOver-Threshold (TOT) approach is adopted for the signal readouts in order to utilize the excellent time resolution of the plastic scintillators. In this paper, we present a method elaborated for establishing a relation between TOT and energy loss.
\end{abstract}

\section{Introduction}

Time-Over-Threshold (TOT) technique is attractive due to the reduced cost and complexity in signal readouts involving the multi-channel systems [1]. In this approach the pulse width of the analog signal gives the estimate of the amplitude of the signal. Thus, TOT technique transforms the charge collection to time measurements and decrease the readout costs significantly by combining the timing and energy information. However, the estimation of energy using the pulse width (timing) suffers a nonlinear relation which in turn depends strongly on the shape of the signal and value of the set threshold [2]. It is reported that applying the multiple thresholds on the signals mitigate the problem of poor linearity to a significant extent [3-5], and it may even enable to reproduce the waveform of the signal $[6,7]$. The Jagiellonian Positron Emission Tomograph (J-PET) is constructed of 192 plastic scintillators attached with photomultipliers at each end [8-12]. Plastic scintillators offer the excellent time resolution which is essential for the time-of-flight (TOF) PET imaging [13] as well to study the decays of positronium atoms [14-16].

In this contribution, we investigate the TOT in the context of the J-PET. The analog signals are probed at four thresholds [17] to attain the better time and energy resolutions, and processed through a trigger-less data acquisition $[18,19]$. The TOT value summed over all thresholds gives the total energy deposition. In the plastic scintillator, the annihilation photons interact predominantly via Compton scatterings and they deposit only partial energy in dependence of their scattering angle. So there is no direct access to the energy of the interacting photons based only on the measured TOT values. In addition, due to the strong non-linear relationship between these two quantities it is of utmost importance to develop a relation among them. Such a relationship can be achieved by performing the studies on the scattering of photons. The geometrical placements of the scintillator strips in the J-PET detector and their high 
angular resolution makes such studies feasible. In the next section the approach to establish the relationship between TOT and energy deposition is illustrated in details.

\section{Experimental analysis}

The detailed technical information and the recent results of the J-PET are presented in the works $[8-12,14-16]$. In this work, we present the idea to perform studies on the scatterings of photons. For this a point like source ${ }^{22} \mathrm{Na}$ with an activity of $1 \mathrm{MBq}$ was placed at the center of the small chamber of diameter $\approx 5 \mathrm{~cm}$. The source was sandwiched in the XAD4 porous material, which enhances the probability of the formation of positronium atom significantly [20]. Photons carrying different energies based on their origin (e.g, from direct $e^{+} e^{-}$annihillation, decay from the para-positronium atom, decay from the ortho-positronium atom and deexcitation photons) are registered via Compton scattering in the plastic scintillators. Large geometrical acceptance of J-PET enables to study the interactions of photons and their respective scattering angles. Fig. 1 presents the experimental setup 1(a) whereas the positioning of source and placement of XAD4 porous material is shown in the adjacent insets. A schematic view of the scattering of photon originating from the center is shown

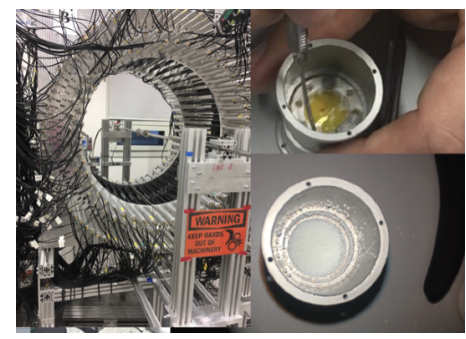

(a)

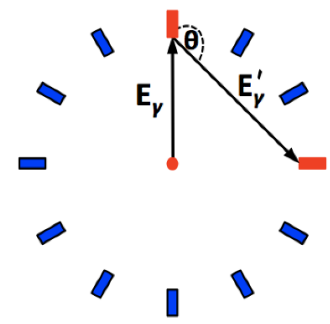

(b)

Figure 1. Left panel shows the J-PET prototype with 3 layers along with the placement of small chamber at the center of detector with ${ }^{22} \mathrm{Na}$ source inside surrounded by XAD4 porous material. Right panel shows the schematic of the photon's interaction with scintillator and the corresponding scattering angle $\theta$.

in Figure 1(b), where the red filled circle is showing the position of the source emitting a photon $\left(\mathrm{E}_{\gamma}\right)$ which interacts with the scintillator and gets scattered $\left(\mathrm{E}_{\gamma}^{\prime}\right)$ at an angle $\theta$. Both scintillators in which the photon interacts are shown in red color. The scattering angles can be calculated using the dedicated offline data analysis framework [21, 22] within a precision of few degrees along with the other hit characteristics (e.g, hit-position, hit-time, TOT), where hit refers to the interaction of photon inside the plastic strip. In order to estimate the energy deposition in such interactions, it is important to know the incident energy of the photon. For the identification of incident photons an algorithm was developed which allow efficiently the selection of photons with different energies (e.g., $511 \mathrm{keV}$ photons from $e^{+} e^{-}$annihilation or from decay of para-positronium atom into 2 photons) and higher energetic 1274.6 $\mathrm{keV}$ photons which are also emitted from the ${ }^{22} \mathrm{Na}$ source. For the precise estimation of the energy deposition the crucial part is to calculate the scattering angle of photon which was validated based on the scatter test $(\mathrm{S})$. The $\mathrm{S}$-test is based on the deviation between measured hit-time [6] difference of two considered hits and calculated time using the distance between measured hit-positions $[8,23]$ divided by the speed of light. Only those scatterings and respective angles are taken into account for which the S-test results in the close proximity to 
zero. For a registered hit, we measured the TOT value and the information on primary and scattered hit allow to access the scattering angles and hence to estimate the energy deposition [24]. Thus, a one-to-one relationship between measured TOT and calculated energy deposition can be achieved with the presented scheme.

\section{Summary}

J-PET detector is composed of 192 plastic scintillators. For the determination of energy deposition of annihilation photons in the scintillators a TOT method is used. In this work, we illustrated the method to develop a relationship between TOT and energy deposition. Such a relationship is very crucial for the tests on the discrete symmetries and measurements of the entanglement properties of the gammas resulting from the positronium decay.

\section{Acknowledgements}

This work was supported by The Polish National Center for Research and Development through grant INNOTECH-K1/IN1/64/159174/NCBR/12, the Foundation for Polish Science through the MPD and TEAM/2017-4/39 programmes, the National Science Centre of Poland through grants no. 2016/21/B/ST2/01222, 2017/25/N/NZ1/00861, the Ministry for Science and Higher Education through grants no. 6673/IA/SP/2016, 7150/E- 338/SPUB/2017/1, 7150/E-338/M/2017, 7150/E-338/M/2018 and the Austrian Science Fund FWF-P26783.

\section{References}

[1] I. Kipnis et al., IEEE Trans. Nucl. Sci. 44, 289 (1997)

[2] T. Orita et al., Nucl. Instr. Meth. A 648, S24 (2011)

[3] H. Kim et al., Nucl. Instr. Meth. A 602, 618 (2009)

[4] L. Njejimana et al. IEEE Trans. Nucl. Sci. 60, 3633 (2008)

[5] M. D. Rolo et al. Jour. Instr. 8, C02050 (2013)

[6] L. Raczyński et al., Nucl. Instr. Meth. A 764, 186-192 (2014)

[7] L. Raczyński et al., Nucl. Instr. Meth. A 786, 105 (2015)

[8] P. Moskal et al., Nucl. Instr. Meth. A 764, 317 (2014)

[9] P. Moskal et al., Nucl. Instr. Meth. A 775, 54 (2015)

[10] P. Moskal et al., Phys. Med. Bio. 61, 2025 (2016)

[11] S. Niedźwiecki et al., Acta Phys. Pol. B 48, 1567 (2017)

[12] P. Kowalski et al., Phys. Med. Bio. 63, 165008 (2018)

[13] C. Casella et al., Nucl. Instr. Meth. A 736161 (2013)

[14] P. Moskal et al., Acta Phys. Polon. B 47, 509 (2016)

[15] B. C. Hiesmayr and P. Moskal, Scientific Reports 7, 15349 (2017)

[16] M. Nowakowski and D. Bedoya Fierro, Acta. Phys. Polon. B 48, 1955 (2017)

[17] M. Palka, Jour. of Instr. 12, P08001 (2017)

[18] G. Korcyl et al., Acta Phys. Polon. B 47, 491 (2016)

[19] G. Korcyl et al., IEEE Trans. on Med. Imag. DOI: 10.1109/TMI.2018.2837741, (2018)

[20] B. Jasinska et al., Acta Phys. Polon. B 47, 453(2016)

[21] W. Krzemień et al., Nukleonika 60, 745 (2015)

[22] W. Krzemień et al., Acta Phys. Polon. A 127, 1491 (2015)

[23] N. G. Sharma et al., Nukleonika 60, 765-769 (2015)

[24] Arthur H. Compton et al. Phys. Rev. 21, 483 (1923) 